DOI: $10.15593 / 2499-9873 / 2020.1 .02$

УДК 517.977 .52

\title{
Г.Ш. Рамазанова
}

Институт систем управления НАН Азербайджана, Баку, Азербайджанская Республика

\section{ЛИНЕАРИЗОВАННЫЕ НЕОБХОДИМЫЕ УСЛОВИЯ ОПТИМАЛЬНОСТИ В ОДНОЙ НЕГЛАДКОЙ ЗАДАЧЕ УПРАВЛЕНИЯ СИСТЕМАМИ ГУРСА - ДАРБУ}

\begin{abstract}
В классе измеримых (в смысле Лебега) и ограниченных управляющих вектор-функций рассматривается одна негладкая задача оптимального управления системой Гурса - Дарбу с многоточечным функционалом качества, являющаяся обобщением терминального типа функционала. Применяя один модифицированный вариант метода приращений и предполагая что правая часть уравнения и функционал качества по вектору состояния имеют производные по любому направлению, доказали необходимое условие оптимальности в терминах производной по направлению, носящее довольно общий характер. Рассмотрен случай квазидифференцируемого функционала качества. В частности, изучена задача минимакса. В предположении выпуклости области управления, с учетом свойств недифференцируемых функций, установлено необходимое условие оптимальности, являющееся аналогом линеаризованного интегрального принципа максимина, имеющее конструктивный характер и обобщающее поточечный линеаризованный (дифференциальный) принцип максимума.

Ключевые слова: негладкая задача оптимального управления системами Гурса - Дарбу, производная по направлению, квазидифференцируемый функционал, задача на минимакс, выпуклая область управления, линеаризованный интегральный принцип максимина, дифференциальный принцип максимума.
\end{abstract}

\section{G.Sh. Ramazanova}

Institute of Control Systems of Azerbaijan NAS, Baku, Republic of Azerbaijan

\section{LINEARIZED REQUIRED OPTIMALITY CONDITIONS IN ONE SMOOTH GURSA-DARBU SYSTEM MANAGEMENT PROBLEM}

In class measurable (in Lebesgue sense) and bounded control vector functions, we consider one non-smooth optimal control problem of the Goursat - Darboux system with a multipoint quality functional, which is a generalization terminal type functional. Applying one modified version of the increment method, and assuming that the right side of the equation and the functional qualities in a vector state have derivatives in any direction, the necessary optimality condition in derivative terms in the direction flax general is proved. The case of a quasidifferentiable quality functional is considered. In particular, the minimax problem is studied. Under the assumption that the control region is convex, taking into account the properties of non-differentiable functions, the necessary optimality condition is established, 
which is an analog of the linearized integral principle of maximin, which is constructive in nature and generalizes the point wise linearized (differential) maximum principle.

Keywords: non-smooth problem of optimal control of Goursat-Darboux systems, directional derivative, quasidifferentiable functional, minimax problem, convex control domain, linearized integral maximin principle, differential maximum principle.

\section{Введение}

В работах [1-5] и других изучались различные задачи оптимального управления, описываемые обыкновенными дифференциальными уравнениями, содержащие негладкие функции. Эти функции могут задавать критерии качества, а также быть правой частью системы дифференциальных уравнений по вектору состояния.

В предлагаемой работе изучается задача оптимального управления системами Гурса - Дарбу с многоточечным негладким функционалом качества в предположении, что правая часть уравнения по вектору состояния и по вектору управления имеет производные по любому направлению, а также удовлетворяет условию Липшица по этим аргументам. При этом функционал качества также считается негладким. В случае выпуклости области управления получено необходимое условие оптимальности первого порядка в терминах производных по направлениям. Отдельно изучен случай квазидифференцируемого функционала. В задаче на минимакс установлено необходимое условие оптимальности типа линеаризованного интегрального принципа максимина, обобщающее и усиливающее линеаризованный принцип максимума.

\section{1. Постановка задачи}

Пусть управляемый процесс на заданном прямоугольнике $D=\left[t_{0}, t_{1}\right] \times\left[x_{0}, x_{1}\right]$ описывается системой нелинейных гиперболических уравнений

$$
z_{t x}=f(t, x, z, u),(t, x) \in D
$$

с краевыми условиями Гурса:

$$
\begin{gathered}
z\left(t_{0}, x\right)=a(x), \quad x \in X=\left[x_{0}, x_{1}\right], \\
z\left(t, x_{0}\right)=b(t), \quad t \in T=\left[t_{0}, t_{1}\right], \\
a\left(x_{0}\right)=b\left(t_{0}\right) .
\end{gathered}
$$


Здесь $f(t, x, z, u)$ - заданная непрерывная в $\Omega=D \times R^{n} \times R^{r} n$-мерная вектор-функция, причем каждая компонента ее удовлетворяет по $P(z, u)^{\prime}$ условию Липшица и имеет производные по любому направлению в пространстве $R^{n} \times R^{r}$, т.е. существуют конечные пределы $\frac{\partial f_{i}(t, x, z, u)}{\partial[h, q]}=\lim _{\alpha \rightarrow+0} \frac{1}{\alpha}\left[f_{i}(t, x, z+\alpha h, u+\alpha q)-f_{i}(t, x, z, u)\right], \quad i=\overline{1, n} ;$
$a(x)$ и $b(t)$ - заданные на $\left[x_{0}, x_{1}\right]$ и $\left[t_{0}, t_{1}\right]$ соответственно $n$-мерные вектор-функции, удовлетворяющие условию Липшица; $u(t, x)$ $r$-мерная измеримая и ограниченная управляющая вектор-функция со значениями из заданного непустого, ограниченного и выпуклого множества $U$, т.е.

$$
u(t, x) \in U \subset R^{r},(t, x) \in D .
$$

Такие управляющие функции назовем допустимыми управлениями.

Как и в работах $[6,7]$, рассматриваются абсолютно непрерывные решения краевой задачи (1)-(2), соответствующие всевозможным допустимым управлениям.

Теоремы существования и единственности абсолютно непрерывного решения краевой задачи (1)-(2) можно найти, например, в работах [6, 7].

Задача заключается в минимизации функционала

$$
S(u)=\Phi\left(z\left(T_{1}, X_{1}\right), \ldots, z\left(T_{k}, X_{k}\right)\right)
$$

при ограничениях (1)-(3).

Здесь $\Phi\left(z_{1}, \ldots, z_{k}\right)$ - заданная в $R^{n}$ скалярная функция, удовлетворяющая условию Липшица и имеющая производные по любому направлению; $T_{i}, X_{i}, \quad i=\overline{1, k},-$ заданные числа, причем $t_{0}<T_{1}<\ldots<T_{k} \leq t_{1}, \quad x_{0}<X_{1}<X_{2}<\ldots<X_{k} \leq x_{1}$.

Допустимое управление $u(t, x)$, доставляющее минимум функционалу (4) при ограничениях (1)-(3), назовем оптимальным управлением, а соответствующий процесс $(u(t, x), z(t, x))$-оптилальным процессом. 


\section{2. Необходимые условия минимума}

Установим необходимые условия минимума в задаче (1)-(4).

Положим

$$
\Delta u_{\varepsilon}(t, x)=\varepsilon(v(t, x)-u(t, x))=\varepsilon q(t, x),
$$

где $q(t, x)=(v(t, x)-u(t, x)), \varepsilon \in[0,1]$ - произвольное число; $u(t, x)-$ заданное допустимое управление, а $v(t, x) \in U-$ произвольное допустимое управление.

В дальнейшем будут использованы обозначения

$$
\frac{\partial f(t, x)}{\partial[h, q]} \equiv \frac{\partial f(t, x, z(t, x), u(t, x))}{\partial[h, q]}, \frac{\partial f(t, x)}{\partial z} \equiv \frac{\partial f(t, x, z(t, x), u(t, x))}{\partial z} .
$$

Пусть $u(t, x ; \varepsilon)=u(t, x)+\varepsilon q(t, x), \quad z(t, x ; \varepsilon)=z(t, x)+\Delta z(t, x ; \varepsilon)$, где $\Delta z(t, x ; \varepsilon)$ - специальное приращение состояния $z(t, x)$, отвечающее специальному приращению (5) управления $u(t, x)$.

Ясно, что специальное приращение состояния будет решением двумерного интегрального уравнения типа Вольтерра:

$$
\begin{gathered}
z(t, x ; \varepsilon)=a(x)+b(t)-a\left(x_{0}\right)+ \\
+\int_{t_{0}}^{t} \int_{x_{0}}^{x} f(\tau, s, z(\tau, s ; \varepsilon), u(\tau, s ; \varepsilon)) d s d \tau .
\end{gathered}
$$

Используя уравнения (5) и (6), в силу условий, наложенных на правую часть уравнения (1), по аналогии со схемами, предложенными в работах [2-5], нетрудно показать, что

$$
z(t, x ; \varepsilon)=z(t, x)+\varepsilon h(t, x)+\mathrm{o}_{1}(\varepsilon ; t, x),
$$

где по определению

$$
h(t, x)=\lim _{\varepsilon \rightarrow 0} \frac{z(t, x ; \varepsilon)-z(t, x)}{\varepsilon},
$$

a $h(t, x)$ является решением краевой задачи 


$$
\begin{gathered}
h_{t x}(t, x)=\frac{\partial f(t, x)}{\partial[h, q]}, \\
h\left(t_{0}, x\right)=0, \quad x \in\left[x_{0}, x_{1}\right], \quad h\left(t, x_{0}\right)=0, \quad t \in\left[t_{0}, t_{1}\right] .
\end{gathered}
$$

Поскольку по предположению функция $\Phi(z)$ дифференцируема по любому направлению и удовлетворяет условию Липшица, с учетом уравнения (7) имеем

$$
\begin{gathered}
S(u(t, x ; \varepsilon))-S(u(t, x))= \\
=\Phi\left(z\left(T_{1}, X_{1}\right)+\varepsilon h\left(T_{1}, X_{1}\right)+\mathrm{o}(\varepsilon), \ldots, z\left(T_{k}, X_{k}\right)+\varepsilon h\left(T_{k}, X_{k}\right)+\mathrm{o}(\varepsilon)\right)- \\
-\Phi\left(z\left(T_{1}, X_{1}\right), \ldots, z\left(T_{k}, X_{k}\right)\right)=\varepsilon \sum_{i=1}^{k} \frac{\partial \Phi\left(z\left(T_{1}, X_{1}\right), \ldots, z\left(T_{k}, X_{k}\right)\right)}{\partial h\left(T_{i}, X_{i}\right)}+\mathrm{o}(\varepsilon) .
\end{gathered}
$$

Из полученного разложения следует, что вдоль оптимального процесса $(u(t, x), z(t, x))$

$$
\varepsilon \sum_{i=1}^{k} \frac{\partial \Phi\left(z\left(T_{1}, X_{1}\right), \ldots, z\left(T_{k}, X_{k}\right)\right)}{\partial h\left(T_{i}, X_{i}\right)}+\mathrm{o}(\varepsilon) \geq 0 .
$$

Из последнего неравенства в силу произвольности $\varepsilon$ вытекает следующий результат.

Теорема 1. Для того чтобы допустимое управление $u(t, x)$ было оптимальным, необходимо, чтобы неравенство

$$
\sum_{i=1}^{k} \frac{\partial \Phi\left(z\left(T_{1}, X_{1}\right), \ldots, z\left(T_{k}, X_{k}\right)\right)}{\partial h\left(T_{i}, X_{i}\right)} \geq 0
$$

выполнялось для всех допустимых вариаций $h\left(T_{i}, X_{i}\right)$ состояния $z\left(T_{i}, X_{i}\right), i=\overline{1, k}$.

Чтобы получить из неравенства (11) конструктивно проверяемые необходимые условия оптимальности, следует использовать специфические свойства функций $f(t, x, z, u)$ и $\Phi(z)$.

Предположим, что $f(t, x, z, u)$ гладкая, т.е. непрерывно дифференцируемая по вектору состояния и по вектору управления функция. Тогда краевая задача Гурса - Дарбу (9)-(10) принимает вид 


$$
\begin{gathered}
h_{t x}(t, x)=\frac{\partial f(t, x)}{\partial z} h(t, x)+\frac{\partial f(t, x)}{\partial u}(v(t, x)-u(t, x)), \\
h\left(t_{0}, x\right)=0, \quad x \in\left[x_{0}, x_{1}\right] \\
h\left(t, x_{0}\right)=0, \quad t \in\left[t_{0}, t_{1}\right] .
\end{gathered}
$$

Пусть $\Phi(z)$ - квазидифференцируемая в точке $z\left(t_{1}, x_{1}\right)$ скалярная функция. Используя определение квазидифференцируемой функции (см., например, работы $[3,5,8]$ ), неравенству (11) можно придать вид

$$
\begin{gathered}
\sum_{i=1}^{k} \frac{\partial \Phi\left(z\left(T_{1}, X_{1}\right), \ldots, z\left(T_{k}, X_{k}\right)\right)}{\partial h\left(T_{i}, X_{i}\right)}= \\
=\sum_{i=1}^{k} \max _{A_{i} \in \underline{\partial} \Phi\left(z\left(T_{i}, X_{i}\right)\right)} A_{i}^{\prime} h\left(T_{i}, X_{i}\right)+\sum_{i=1}^{k} \min _{B_{i} \in \bar{\partial} \Phi\left(z\left(T_{i}, X_{i}\right)\right)} B_{i}^{\prime} h\left(T_{i}, X_{i}\right) \geq 0 .
\end{gathered}
$$

Здесь $\left[\underline{\partial} \Phi\left(z\left(T_{i}, X_{i}\right)\right), \bar{\partial} \Phi\left(z T_{i}, X_{i}\right)\right]$ - квазидифференциал функции $\Phi(z)$ в точке $z\left(T_{i}, X_{i}\right)$.

Применяя формулу для решения краевой задачи Гурса линейного неоднородного гиперболического уравнения с негладкими коэффициентами (см., например, работу [9]), представим решение краевой задачи (12)-(13) в виде

$$
h(t, x)=\int_{t_{0}}^{t} \int_{x_{0}}^{x} R(t, x ; \tau, s) \frac{\partial f(\tau, s)}{\partial u}(v(\tau, s)-u(\tau, s)) d s d \tau,
$$

где $R(t, x ; \tau, s)-(n \times n)$-матричная функция, являющаяся решением уравнения

$$
R(t, x ; \tau, s)=E+\int_{\tau}^{t} \int_{s}^{x} R(t, x ; \alpha, \beta) \frac{\partial f(\tau, s)}{\partial z} d s d \tau,
$$

а $E$ - единичная $(n \times n)$-матрица.

Пусть $\alpha_{i}(t, x), \quad i=\overline{1, k}$ - характеристическая функция прямоугольника $\left[t_{0}, T_{i}\right] \times\left[x_{0}, X_{i}\right], i=\overline{1, k}$. Тогда из представления (15) получаем, что 


$$
h\left(T_{i}, X_{i}\right)=\int_{t_{0}}^{t_{1}} \int_{x_{0}}^{x_{1}} \alpha_{i}(t, x) R\left(T_{i}, X_{i}, \tau, s\right) \frac{\partial f(\tau, s)}{\partial u}(v(\tau, s)-u(\tau, s)) d s d \tau
$$

Из неравенства (14) имеем

$$
\begin{gathered}
\sum_{i=1}^{k} \frac{\partial \Phi\left(z\left(T_{i}, X_{i}\right)\right)}{\partial h\left(T_{i}, X_{i}\right)}= \\
=\sum_{i=1}^{k} \max _{A_{i} \in \underline{\partial} \Phi\left(z\left(T_{i}, X_{i}\right)\right)} \int_{t_{0}}^{t_{1}} \int_{x_{0}}^{x_{1}} A_{i}^{\prime} \alpha_{i}(t, x) R\left(T_{i}, X_{i} ; \tau, s\right) \frac{\partial f(\tau, s)}{\partial u}(v(\tau, s)-u(\tau, s)) d s d \tau+ \\
+\sum_{i=1}^{k} \min _{B_{i} \in \bar{\partial} \Phi\left(z\left(T_{i}, X_{i}\right)\right)} \int_{t_{0}}^{t_{1}} \int_{x_{0}}^{x_{1}} B_{i}^{\prime} \alpha_{i}(t, x) R\left(T_{i}, X_{i} ; \tau, s\right) \frac{\partial f(\tau, s)}{\partial u}(v(\tau, s)-u(\tau, s)) d s d \tau \geq 0 .
\end{gathered}
$$

Положим

$$
\begin{gathered}
\psi_{A_{i}}(\tau, s)=R^{\prime}\left(T_{i}, X_{i} ; \tau, s\right) A_{i} \alpha_{i}(t, x), \\
\psi_{B_{i}}(\tau, s)=R^{\prime}\left(T_{i}, X_{i} ; \tau, s\right) B_{i} \alpha_{i}(t, x), \\
H\left(t, x, z(t, x), u(t, x), \psi_{A_{i}}(t, x)\right)=\psi_{A_{i}}^{\prime}(t, x) f(t, x, z(t, x), u(t, x)), \\
H\left(t, x, z(t, x), u(t, x), \psi_{B_{i}}(t, x)\right)=\psi_{B_{i}}^{\prime}(t, x) f(t, x, z(t, x), u(t, x)) .
\end{gathered}
$$

Учитывая обозначения (8) и (9), полученный результат можно сформулировать следующим образом.

теорема 2. Для того чтобы допустимое управление $u(t, x)$ было оптимальныл, необходимо выполнение неравенства

$$
\begin{aligned}
& \sum_{i=1}^{k} \max _{A_{i} \in \underline{\partial} \Phi\left(z\left(T_{i}, X_{i}\right)\right)}\left[\int_{t_{0}}^{t_{1}} \int_{x_{0}}^{x_{1}} H_{u}^{\prime}\left(t, x, z(t, x), u(t, x), \psi_{A_{i}}(t, x)\right)(v(t, x)-u(t, x)) d t d x+\right. \\
& \left.+\sum_{i=1}^{k} \min _{B_{i} \in \bar{\partial} \Phi\left(z\left(T_{i}, X_{i}\right)\right)} \int_{t_{0}}^{T_{i}} \int_{x_{0}}^{X_{i}} H_{i}^{\prime} R\left(t, x, z(t, x), u(t, x), \psi_{B_{i}}(t, x)\right)(v(t, x)-u(t, x)) d t d x\right] \geq 0 \\
& \text { для всех } v(t, x) \in U,(t, x) \in D .
\end{aligned}
$$




\section{3. Линеаризованный интегральный принцип минимакса}

Предположим, что правая часть уравнения (1) непрерывнодифференцируема по $(z, u)$, а функционал качества имеет вид

$$
S(u)=\max _{y \in Y} \varphi\left(z\left(t_{1}, x_{1}\right), y\right) .
$$

Здесь $Y$ - компактное множество векторов, а $\varphi(z, y)$ - непрерывная по совокупности переменных вместе с частными производными по $z$ скалярная функция. Требуется найти минимум функционала (16) при ограничениях (1)-(3), считая гладкой правую часть уравнения (1) и по вектору состояния, и по вектору управления. Таким образом, мы рассматриваем задачу на минимакс.

Предполагаем, что в задаче (1)-(3), (16) существует оптимальное управление.

Пусть $(u(t, x), z(t, x)) \quad-\quad$ фиксированный, $(\bar{u}(t, x)=u(t, x)+\Delta u(t, x), \quad \bar{z}(t, x)=z(t, x)+\Delta z(t, x))-$ произвольный допустимые процессы.

Введем множество максимумов функции $\varphi(z, y)$ (см., например, работу [10]):

$$
Y(z)=\left\{y \in Y: \varphi\left(z\left(t_{1}, x_{1}\right), y\right)=\max _{\bar{y} \in Y} \varphi\left(z\left(t_{1}, x_{1}\right), \bar{y}\right)\right\} .
$$

Нетрудно показать, что приращение $\Delta z(t, x)$ состояния $z(t, x)$ является решением линеаризованной задачи

$$
\begin{gathered}
\Delta z_{t x}(t, x)=f_{z}(t, x, z(t, x), u(t, x)) \Delta z(t, x)+ \\
+f_{u}(t, x, z(t, x), u(t, x)) u(t, x)+\mathrm{o}_{1}(\|\Delta z(t, x)\|+\|\Delta u(t, x)\|) .
\end{gathered}
$$

Здесь норма вектора $a=\left(a_{1}, a_{2}, \ldots, a_{n}\right)^{\prime}$ задается формулой $\|a\|=\sum_{i=1}^{n}\left|a_{i}\right|$

$$
\Delta z\left(t_{0}, x\right)=0, x \in\left[x_{0}, x_{1}\right], \Delta z\left(t, x_{0}\right)=0, t \in\left[t_{0}, t_{1}\right]
$$


a $\mathrm{O}_{1}(\alpha)$ (величина более высокого порядка малости, чем $\alpha$ ) определяется из разложения

$$
\begin{gathered}
f(t, x, \bar{z}(t, x), \bar{u}(t, x))+f(t, x, z(t, x), u(t, x))= \\
=f_{z}(t, x, z(t, x), u(t, x)) \Delta z(t, x)+ \\
+f_{u}(t, x, z(t, x), u(t, x)) u(t, x)+\mathrm{o}_{1}(\|\Delta z(t, x)\|+\|\Delta u(t, x)\|) .
\end{gathered}
$$

Считая $\psi_{y}(t, x)$ произвольной $n$-мерной вектор-функцией, введем обозначение $H\left(t, x, z, u, \psi_{y}\right)=\psi_{y}^{\prime} \cdot f(t, x, z, u)$, с учетом которого получим

$$
\begin{gathered}
\int_{t_{0}}^{t_{1}} \int_{x_{0}}^{x_{1}} \psi_{y}^{\prime}(t, x) \Delta z(t, x) d t d x= \\
=\int_{t_{0}}^{t_{1} x_{x_{0}}}\left[H\left(t, x, \bar{z}(t, x), \bar{u}(t, x), \psi_{y}(t, x)\right)-\right. \\
\left.-H\left(t, x, z(t, x), u(t, x), \psi_{y}(t, x)\right)\right] d t d x .
\end{gathered}
$$

Так как выполнены краевые условия (17), то

$$
\int_{t_{0}}^{t_{1}} \int_{x_{0}}^{x_{1}} \psi_{y}^{\prime}(t, x) \Delta z(t, x) d t d x=\psi_{y}^{\prime}(t, x) \Delta z(t, x)-\int_{t_{0}}^{t_{1}} \int_{x_{0}}^{x_{1}} \frac{\partial^{2} \psi_{y}^{\prime}(t, x)}{\partial t \partial x} \Delta z(t, x) d t d x .
$$

Из оценок, приведенных в работах $[11,12]$, следует, что

$$
\|\Delta z(t, x)\| \leq L_{1} \int_{t_{0}}^{t_{1}} \int_{x_{0}}^{x_{1}}\|\Delta u(t, x)\| d t d x
$$

где $L_{1}-$ некоторая положительная константа.

Специальное приращение допустимого управления $u(t, x)$ определим по формуле

$$
\Delta u(t, x: \mu)=\mu(v(t, x)-u(t, x)),
$$

где $\mu \in[0,1]$ - произвольное число, а $v(t, x) \in U,(t, x) \in D$ - произвольное допустимое управление. 
Через $\Delta z(t, x: \mu)$ обозначим специальное приращение состояния $z(t, x)$, отвечающее специальному приращению управления.

Из оценки неравенства (19) следует, что $\|\Delta z(t, x: \mu)\|$ имеет порядок малости $\mu$. Таким образом, принимая во внимание формулу (18) и применяя формулу Тейлора, по схеме, аналогичной схеме из работ $[1,3,12]$, специальное приращение функционала качества можно записать в виде

$$
\begin{gathered}
\Delta S_{\mu}(u)=S(u(t, x)+\Delta u(t, x: \mu))-S(u(t, x))= \\
=\max _{y \in y(z)} \int_{t_{0}}^{t_{1}} \int_{x_{0}}^{x_{1}}\left[\frac{\partial \varphi^{\prime}\left(z\left(t_{1}, x_{1}\right), y\right)}{\partial z} \Delta z\left(t_{1}, x: \mu\right)+\right. \\
+\psi_{y}^{\prime}\left(t_{1}, x_{1}\right) \Delta z\left(t_{1}, x_{1}: \mu\right)-\int_{t_{0}}^{t_{1} x_{1}} \frac{\partial^{2} \psi_{y}^{\prime}(t, x)}{\partial t \partial x} \Delta z(t, x: \mu) d t d x- \\
-\int_{t_{0}}^{t_{1} x_{0}} \int_{1}^{x_{1}} \frac{\partial H^{\prime}\left(t, x, z(t, x), u(t, x), \psi_{y}(t, x)\right)}{\partial z} \Delta z(t, x: \mu) d t d x- \\
-\mu \int_{t_{0}}^{t_{1}} \frac{\partial H_{0}^{\prime}\left(t, x, z(t, x), u(t, x), \psi_{y}(t, x)\right)}{\partial u} \times \\
\left.\times(v(t, x)-u(t, x)) d t d x+\mathrm{o}_{y}(\mu)\right] d t d x .
\end{gathered}
$$

Если предполагать, что $\psi_{y}(t, x)$ является решением краевой задачи (сопряженная система)

$$
\begin{gathered}
\frac{\partial^{2} \psi_{y}(t, x)}{\partial t \partial x}=\frac{\partial H\left(t, x, z(t, x), u(t, x), \psi_{y}(t, x)\right)}{\partial z} \\
\psi_{y}\left(t_{1}, x_{1}\right)=-\frac{\partial \varphi^{\prime}\left(z\left(t_{1}, x_{1}\right), y\right)}{\partial z}
\end{gathered}
$$

то разложение (20) примет вид

$$
\begin{aligned}
\Delta S_{\mu}(u)=- & \mu \min _{y \in Y(z)} \int_{t_{0}}^{t_{1}} \int_{x_{0}} \frac{\partial H^{\prime}\left(t, x, z(t, x), u(t, x), \psi_{y}(t, x)\right)}{\partial u} \times \\
& \times(v(t, x)-u(t, x)) d t d x+o(\mu) .
\end{aligned}
$$


В силу достаточной малости $\mu$ из разложения (21) выводим следующее утверждение.

Теорема 3. Для оптимальности допустимого управления $u(t, x)$ в задаче (1)-(3), (21) необходимо, чтобы неравенство

$$
\min _{y \in Y(z)} \int_{t_{0}}^{t_{1}} \int_{x_{0}}^{x_{1}} \frac{\partial H^{\prime}\left(t, x, z(t, x), u(t, x), \psi_{y}(t, x)\right)}{\partial u}(v(t, x)-u(t, x)) d t d x \leq 0
$$

выполнялось для всех $v(t, x) \in U,(t, x) \in D$.

Доказанная теорема является аналогом линеаризованного интегрального принципа максимина [13], но, в отличие от гладкого критерия качества, не является следствием линеаризованного условия максимума [5].

\section{Заключение}

В работе изучается одна негладкая задача оптимального управления системами гиперболических уравнений с краевыми условиями Гурса. При различных предположениях установлен ряд необходимых условий оптимальности первого порядка. Отдельно изучен случай квазидифференцируемого функционала качества. Установлен аналог линеаризованного интегрального принципа максимина.

\section{Список литературы}

1. Габасов Р., Кириллова Ф.М. Принцип максимума в теории оптимального управления. - М.: ЛИБРОКОМ, 2011. - 272 с.

2. Демьянов В.Ф., Никулина В.Н., Шаблинская И.Р. Задача оптимального управления негладкими дифференциальными связями // Дифференциальные уравнения. - 1985. - Т. 21, № 8. - С. 1324-1330.

3. Негладкие задачи теории оптимизации и управления / В.Ф. Демьянов, Т.К. Виноградова, В.Н. Никулина [и др.]; под ред. В.Ф. Демьянова. - Л.: Изд-во ЛГУ, 1982. - $322 \mathrm{c}$.

4. Виноградова Т.К., Демьянов В.Ф. К необходимым условиям в минимаксных задачах управления // Журнал вычислительной математики и математической физики. - 1974. - Т. 12, № 1. - С. 232-236.

5. Демьянов В.Ф., Рубинов А.М. Основы негладкого анализа и квазидифференциальное исчисление. - М.: Наука, 1990. - 432 с. 
6. Плотников В.И., Сумин В.И. Оптимизация объектов с распределенными параметрами, описываемыми системами Гурса - Дарбу // Журнал вычислительной математики и математической физики. - 1972. - Т. 12, № 1. C. 61-77.

7. Гасанов К.К., Гасанова Л.К. Теорема существования и единственности для нелинейных систем Гурса - Дарбу // Вестник Бакинского университета. Сер. физ.-мат. наук. - 2012. - № 4. - С. 37-44.

8. Демьянов В.Ф., Васильев Л.В. Недифференцируемая оптимизация. М.: Наука, 1981. - 384 с.

9. Ахиев С.С., Ахмедов К.Т. Об интегральном представлении решений некоторых систем дифференциальных уравнений // Известия АН Азерб. ССР. Сер. физ.-техн. и матем. наук. - 1973. - № 2. - С. 116-120.

10. Демьянов В.Ф. Минимакс. Дифференцируемость по направлениям. - Л.: Изд-во ЛГУ, 1974. - 185 с.

11. Егоров А.И. Об оптимальном управлении процессами в некоторых системах с распределенными параметрами // Автоматика и телемеханика. 1964. - Т. 25, вып. 5. - С. 613-623.

12. Васильев О.В., Срочко В.А., Терлецкий В.А. Методы оптимизации и их приложения. Ч. 2. Оптимальное управление / под ред. А.П. Меренкова. Новосибирск: Наука: Сиб. отд-ние, 1990. - 148 с.

13. Альсевич В.В. Необходимые условия оптимальности для минимаксных задач оптимизации // Дифференциальные уравнения. -1976. - Т. 12, № 8. - С. 1384-1391.

\section{References}

1. Gabasov R., Kirillova F.M. Printsip maksimuma v teorii optimal'nogo upravleniia [Maximum principle in optimal control theory]. Moscow, LIBROKOM, 2011, 272 p.

2. Dem'ianov V.F., Nikulina V.N., Shablinskaia I.R. Zadacha opti-mal'nogo upravleniia negladkimi differentsial'nymi sviaziami [Problem of optimal control of non-smooth differential connections]. Differential Equations, 1985, vol. 21, iss. 8, pp. 1324-1330.

3. Dem'ianov V.F., Vinogradova T.K., Nikulina V.N. and others. Negladkie zadachi teorii optimizatsii i upravleniia [Non-smooth optimization and control theory problems]. Leningrad, Izdatel'stvo Leningradskogo gosudarstvennogo universiteta. 1982, $322 \mathrm{p}$.

4. Vinogradova T.K. Dem'ianov V.F. Necessary conditions in control problems of the minimax type. USSR Computational Mathematics and Mathematical Physics, 1974, vol. 14, iss. 1, pp.232-236. 
5. Dem'ianov V.F., Rubinov A.M. Osnovy negladkogo analiza i kvazidifferentsial'noe ischislenie [Foundations of non-smooth analysis and quasidifferential calculus]. Moscow, Nauka. 1990. 432 p.

6. Plotnikov V.I., Sumin V.I. The optimization of objects with distributed parameters, described by Goursat-Darboux systems. USSR Computational Mathematics and Mathematical Physics, 1972, vol.12, iss. 1, pp. 73-92.

7. Gasanov K.K., Gasanova L.K. Teorema sushchestvovaniia i edinstvennosti dlia nelineinykh sistem Gursa-Darbu [Optimization of objects with distributed parameters described by systems of Goursat-Darboux equations]. Vestnik Bakinskogo universiteta. Ser. fiz. mat. Nauk, 2012, no. 4, pp.37-44.

8. Dem'ianov V.F., Vasil'ev L.V. Nedifferentsiruemaia optimizatsiia [Undifferentiated optimization]. Moscow, Nauka. 1981, 384 p.

9. Akhiev S.S., Akhmedov K.T. Ob integral'nom predstavlenii reshenii nekotorykh sistem differentsial'nykh uravnenii [On the integral representation of solutions of some systems of differential equations]. Izv. AN Az. SSR. Ser. fiz.tekhn. i mat. nauk, 1973, vol. 2, pp.116-120.

10. Dem'ianov V.F. Minimaks. Differentsiruemost' po napravleniiam [Minimax. Differentiability in the directions]. Leningrad. Izdatel'stvo Leningradskogo gosudarstvennogo universiteta, 1974, $185 \mathrm{c}$.

11. Egorov A.I. Ob optimal'nom upravlenii protsessami v nekotorykh sistemakh s raspredelennymi parametrami [On the optimal control of processes in some systems with distributed parameters]. Automation and remote control. 1964, vol. 5, pp. 613-623.

12. Vasil'ev O.V., Srochko V.A., Terletskii V.A. Metody optimizatsii i ikh prilozheniia, Ch2. Optimal'noe upravlenie [Optimization methods and their applications, Part 2. Optimal control]. Novosibirsk. Nauka. 1990, 148 p.

13. Al'sevich V.V. Neobkhodimye usloviia optimal'nosti dlia minimaksnykh zadach optimizatsii [Necessary optimality conditions for minimax optimization problems]. Differential Equations. 1976, vol. 12, iss. 8, pp.1384-1391.

Получено 19.09.2019

Принято 11.02.2020

\section{Сведения об авторе}

Рамазанова Говхар Шабан гызы (Баку, Азербайджанская Республика) - научный сотрудник и диссертант, Институт систем управления НАН Азербайджана (Az 1141, Баку, ул. Б. Вагабзаде, 68, e-mail: akja@rambler.ru). 


\section{About the author}

Govkhar Sh. Ramazanova aban (Baku, Republic of Azerbaijan) - Researcher, Institute of Control Systems of Azerbaijan NAS (Az 1141, Baku, Bakhtiyar Vahabzadeh st., 68, e-mail: akja@rambler.ru).

Библиографическое описание статьи согласно ГОСТ Р 7.0.100-2018:

Рамазанова, Г. Ш. Линеаризованные необходимые условия оптимальности в одной негладкой задаче управления системами Гурса - Дарбу / Г. Ш. Рамазанова. DOI 10.15593/2499-9873/2020.1.02. - Текст : непосредственный // Прикладная математика и вопросы управления = Applied Mathematics and Control Sciences. - 2020. № 1. - С. 20-33.

\section{Цитирование статьи в изданиях РИНЦ:}

Рамазанова Г.Ш. Линеаризованные необходимые условия оптимальности в одной негладкой задаче управления системами Гурса - Дарбу // Прикладная математика и вопросы управления. - 2020. - № 1. - С. 20-33. DOI: 10.15593/2499-9873/2020.1.02

Цитирование статьи в references и международных изданиях:

Cite this article as:

Ramazanova G.Sh. Linearized required optimality conditions in one smooth Goursat-Darboux system management problem. Applied Mathematics and Control Sciences, 2020, no. 1, pp. 20-33. DOI: 10.15593/2499-9873/2020.1.02 (in Russian) 